\title{
Redes de atenção à saúde: uma estratégia para integração dos sistemas de saúde
}

Health care networks: a strategy for health systems integration

Redes de Atención de salud: una estrategia para la integración de los sistemas de salud

\section{Adalvane Nobres DamacenoI, Maria Alice Dias da Silva Lima ${ }^{\text {II }}$, Vanessa Rodrigues PucciII, Teresinha Heck Weiller ${ }^{\mathrm{IV}}$}

Resumo: Objetivo: discutir e refletir sobre as Redes de Atenção à Saúde, seus conceitos teóricos e marcos legais nas políticas de saúde. Método: estudo teórico reflexivo, baseado na literatura científica sobre conceitos teóricos e marcos legais das Redes de Atenção à Saúde. Resultados: a integração dos serviços de saúde é uma resposta à fragmentação do cuidado e possui multiplicidade de conceitos, princípios e dimensões. As Redes de Atenção à Saúde revelam-se como uma estratégia para integração dos serviços e são uma alternativa na qualificação do cuidado. Experiências nacionais e internacionais indicam que o cuidado em rede, garante maior eficácia das ações de saúde com cuidados múltiplos pautados em uma integração sistêmica. Conclusões: a conformação de Redes de Atenção à Saúde colabora para superação do modelo verticalizado. A estruturação é pautada com base no perfil epidemiológico e colabora para melhoria da atenção prestada, com superação de lacunas assistenciais e redução de custos.

Descritores: Atenção Primária à Saúde; Integração de Sistemas, Serviços de Saúde; Cobertura de Serviços de Saúde; Prestação integrada de cuidados de saúde

Abstract: Objective: to discuss and reflect on Health Care Networks, their theoretical concepts and legal frameworks in health policies. Method: a reflective theoretical study, based on the scientific literature on theoretical concepts and legal frameworks of Health Care Networks. Results: the integration of health services is a response to the fragmentation of care, and has a multiplicity of concepts, principles and dimensions. Health Care Networks are revealed as a strategy for the integration of services and are an alternative in the qualification of care. National and international experiences indicate that networked care ensures greater effectiveness of health actions with multiple care based on a systemic integration. Conclusions: the conformation of Health Care Networks contributes to overcoming the vertical model. The structuring is based on the epidemiological profile and contributes to improving the care provided, overcoming care gaps and reducing costs.

\footnotetext{
I Enfermeiro. Mestre em Enfermagem. Universidade Federal do Rio Grande do Sul (UfRGS). Porto Alegre, Rio Grande do Sul, Brasil. adalvanedamaceno@gmail.com ORCID http://orcid.org/0000-0002-4681-0602

${ }^{\text {II }}$ Enfermeira. Doutora em Enfermagem. Universidade Federal do Rio Grande do Sul (UFRGS). Porto Alegre, Rio Grande do Sul, Brasil. malice@enf.ufrgs.br ORCID https://orcid.org/0000-0002-3490-7335

III Nutricionista. Mestra em Enfermagem. Universidade Federal de Santa Maria (UFSM). Santa Maria, Rio Grande do Sul, Brasil. vanessarp@hotmail.com ORCID https://orcid.org/0000-0002-4036-316X

IV Enfermeira. Doutora em Saúde Pública. Universidade Federal de Santa Maria (UFSM). Santa Maria, Rio Grande do Sul, Brasil. weiller2@hotmail.com ORCID https://orcid.org/0000-0003-2531-0155
} 
Redes de atenção à saúde: uma estratégia para integração dos sistemas de saúde $\mid 2$

Descriptors: Primary Health Care; Systems Integration; Health Services Coverage; Delivery of Health Care, Integrated

Resumen: Objetivo: discutir y reflexionar sobre las Redes de Atención de Salud, sus conceptos teóricos y los marcos legales en las políticas de salud. Método: estudio teórico reflexivo, basado en literatura científica sobre conceptos teóricos y marcos legales. Resultados: la integración de los servicios de salud es una respuesta a la fragmentación de la atención y tiene una multiplicidad de conceptos, principios y dimensiones. Las Redes de Atención de Salud son una estrategia para integrar servicios y son una alternativa en la calificación de la atención. Las experiencias nacionales e internacionales indican que la atención en red garantiza una mayor efectividad de las acciones de salud con atención múltiple basada en una integración sistémica. Conclusiones: la conformación de Redes de Atención de Salud contribuye a superar el modelo vertical. La estructura se basa en el perfil epidemiológico y contribuye a mejorar la atención brindada, superar las brechas de atención y reducir los costos.

Descriptores: Atención Primaria de Salud; Integración de Sistemas; Cobertura de los Servicios de Salud; Prestación Integrada de Atención de Salud

\section{Introdução}

A responsabilidade da Atenção Primária à Saúde (APS) nas ações de coordenação de cuidados e garantia da continuidade da atenção, tem sido objeto de discussão e reflexão no campo da saúde. Para o momento sanitário atual, com o crescimento da incidência de doenças crônicas e alteração na estrutura etária da população, revela-se necessária uma resposta dos serviços de saúde para a organização de um sistema de referência responsável, eficiente e integrado. ${ }^{1}$

A Conferência Internacional em Alma-Ata indicou os cuidados primários como parte integrante dos sistemas nacionais de saúde. Assim, constituem a função central e o foco principal no que diz respeito ao desenvolvimento socioeconômico global das comunidades. Devem, entre outras funções, ser apoiados por sistemas de referência integrados, funcionais e mutuamente amparados, levando à progressiva melhoria dos cuidados gerais de saúde para todos, dando prioridade aos que têm mais necessidades. ${ }^{2}$

Novas formas de integração dos serviços de saúde têm sido propostas com base no fortalecimento da APS, principalmente, por meio de seu atributo de coordenação, colocando-a como um eixo estruturante do sistema. $^{3}$ Uma das propostas de integração constitui-se na 
implementação de Redes de Atenção à Saúde (RAS), nas quais a APS assume papel de eixo estruturante no desenho e funcionamento desses sistemas. ${ }^{4}$

As RAS são definidas como os serviços e ações que intervêm em processos de saúdedoença, pautados em diferentes densidades tecnológicas, logísticas e de gestão para assegurar a integralidade do cuidado, melhorar o acesso, a equidade, bem como a eficácia proposta no Sistema Único de Saúde (SUS). ${ }^{4-6}$ Somam-se a isso, os objetivos comuns e ações cooperativas e interdependentes dentro de organizações participativas e democráticas, com conjuntos de serviços de saúde que permitam ofertar uma atenção contínua e integral à determinada população, superando a fragmentação da atenção e da gestão dos serviços de saúde. ${ }^{4-6}$

Frente a esse cenário, as RAS são consideradas a melhor estratégia para o enfrentamento da fragmentação da atenção e dos problemas de saúde pública, ${ }^{7}$ haja vista os modelos de atenção serem diferentes, tanto para as condições crônicas, quanto para as condições agudas. ${ }^{8}$ Embora a conformação de RAS venha sendo enfatizada nas políticas de saúde, existem alguns questionamentos sobre sua efetivação e suas contribuições para o fortalecimento da APS, tais como: de que maneira a dificuldade no acesso aos serviços de saúde pela população pode ser superada? como vencer a fragmentação do cuidado envolvendo gestores, profissionais e usuários dos serviços de saúde?

Considerando essas indagações, este artigo tem como objetivo discutir e refletir sobre as Redes de Atenção à Saúde, seus conceitos teóricos e marcos legais nas políticas de saúde.

\section{Integração dos sistemas de saúde}

A Organização Mundial da Saúde (OMS) conceitua serviços integrados como aqueles preventivos e curativos, ofertados de maneira continuada ao longo do tempo aos usuários, em diferentes níveis de atenção. ${ }^{9}$ A discussão sobre integração dos sistemas de saúde surgiu em 
Redes de atenção à saúde: uma estratégia para integração dos sistemas de saúde $\mid 4$

resposta à fragmentação dos cuidados e pelos obstáculos a serem superados para promover a articulação entre os serviços de saúde..$^{10,11}$

Entretanto, a literatura apresenta uma multiplicidade de conceitos, princípios e dimensões sobre a integração dos sistemas de saúde. Assim, os serviços de saúde integrados respondem pela promoção da saúde, prevenção de doenças, diagnóstico, tratamento, gerenciamento de doenças, reabilitação e cuidados paliativos, de acordo com as necessidades ao longo da vida. Além disso, os responsáveis pela criação e manutenção desses serviços têm a finalidade de coordenar sua interdependência, a fim de que trabalhem em projetos coletivos e coerentes para o financiamento, administração, organização e prestação de serviços. ${ }^{11-13}$

Embora exista uma diversidade de abordagens e estratégias para a integração de sistemas, a literatura identifica dez princípios universais para a integração dos serviços de saúde. Esses princípios abrangem os serviços integrais em todos os níveis do continuum de cuidados, bem como o foco no paciente, a cobertura geográfica, prestação de cuidados padronizados por equipes interprofissionais, gestão de desempenho dos serviços. E, ainda, os sistemas de comunicação, a cultura organizacional, a integração de médicos nas equipes, a estrutura de governança e a gestão financeira e, por fim, elementos que podem ser utilizados por tomadores de decisão visando à integração. ${ }^{14}$

Além dos princípios supracitados, são indicados elementos conceituais e taxonômicos que permitem a compreensão da integração de serviços de saúde, que abrangem o tipo de integração (organizacional, profissional, cultural e tecnológico); o nível em que ocorre a integração (macro, meso e micro); o processo de integração (a forma como a assistência integrada é organizada e gerenciada); a amplitude da integração (a todo um grupo populacional ou a um grupo específico de clientes); e o grau ou a intensidade da integração (através de um continuum que abrange desde ligações informais à coordenação de cuidados mais gerenciados e equipes ou organizações totalmente integradas). ${ }^{12-14}$ 
A aplicação desses conceitos é uma alternativa para a mudança no modelo de cuidado para serviços de saúde eficientes e eficazes que priorizem a APS como cenário de coprodução de saúde. Isso engloba a necessidade de um sistema de referência seguro e integrado. Requer investimento claro em novas formas de cuidado que devem ser coerentes e com regramentos pré-definidos, por exemplo, no caso das condições crônicas que exigem o uso simultâneo de diversos serviços.

\section{Redes de atenção à saúde}

A primeira descrição completa de uma rede regionalizada foi apresentada pelo Relatório Dawson, publicado em 1920. Naquele contexto, almejavam-se mudanças no sistema de proteção social e na organização da provisão de serviços de saúde no Reino Unido, após a Primeira Guerra Mundial, com base na coordenação entre serviços preventivos e curativos. ${ }^{15}$

Para Dawson, o território estabeleceria a organização de serviços, os quais deveriam ofertar a atenção integral para população, com base nas características de "porta de entrada" do sistema. Ademais, consideraria a distribuição da população, os meios de transporte e os fluxos estabelecidos, variando em tamanho e complexidade, segundo as circunstâncias. ${ }^{15,16}$ Os casos que não pudessem ser resolvidos em centros primários e secundários seriam encaminhados a um hospital de referência vinculado a tais serviços. A organização dos profissionais seria de forma integrada, de modo que poderiam acompanhar os indivíduos adscritos desde a queixa inicial até o regresso ao domicílio. ${ }^{16}$

Ao Relatório Dawson coube também, introduzir a territorialização dos sistemas de saúde globais e indicar a necessidade de articulação entre a saúde pública e a atenção individual. No panorama da organização de serviços, formulou os conceitos de níveis de atenção, porta de entrada, vínculo, referência e coordenação pela atenção primária. E, sobretudo, considerou os mecanismos de integração como sistemas de informação e de transportes. ${ }^{16}$ 
Redes de atenção à saúde: uma estratégia para integração dos sistemas de saúde |6

A organização em redes foi concebida como uma resposta para a garantia do acesso e qualidade a toda população, por meio de oferta de serviços e adoção de mecanismos de referência. Portanto, a regionalização deveria ser baseada em territórios compatíveis com autossuficiência em recursos de saúde em todos os níveis de atenção, subdivididos em distritos, sub-regiões ou microrregiões como forma de assegurar o acesso. ${ }^{17,18}$

A conformação de RAS, assim como a sua importância para a qualificação dos sistemas de saúde, se justifica pelo aumento crescente da incidência e prevalência das doenças crônicas, que demandam a construção da integralidade para intervir nos custos crescentes dos sistemas de saúde.,17 Dessa forma, entende-se que a oferta de serviços deve responder à busca das necessidades dos usuários, a partir do olhar ampliado, o que pode beneficiar sua saúde nos seus contatos com os diferentes pontos do sistema.

As propostas de redes no suporte às políticas públicas têm sido crescentemente adotadas para superar o modelo burocrático e hierárquico hegemônico, num contexto de discussões das questões sociais, de processos de privatização, de descentralização acelerada, de globalização, de proliferação de organizações não governamentais e de fortalecimento do controle público. ${ }^{18,19}$ Desse modo, a reorientação de modelos de atenção precisa incluir ações intersetoriais e contemplar discussões sobre os complexos desafios de produção da saúde, com uma compreensão abrangente dos seus problemas de forma regionalizada, articulando políticas para a promoção com foco em um modelo de atenção integral.

\section{Elementos conceituais das Redes de Atenção à Saúde}

As RAS são uma forma de organização das ações e serviços de promoção, prevenção e recuperação da saúde, em todos os níveis de atenção de um determinado território. ${ }^{17}$ Assim, a integralidade do cuidado poderá ser garantida pela construção de tais ações, bem como de serviços de saúde com diferentes densidades tecnológicas e integradas por meio de sistemas de apoio técnico, logístico e de gestão. ${ }^{11}$ Logo, a implementação de serviços de saúde com 
centralidade no usuário e suas necessidades em saúde, deve ser a base para a definição clara dos serviços de referência e fluxos de informação no território.

Em sua conformação estão três elementos indissociáveis: a população, a estrutura operacional e o modelo de atenção à saúde. No que se refere a população, esta é colocada sob sua responsabilidade sanitária e econômica, e deve ser organizada sob forma de gerenciamento, com ênfase à gestão da oferta da atenção à saúde. A estrutura operacional é constituída pelos “nós” das redes e pelas ligações materiais e imateriais que comunicam esses diferentes nós. Entende-se por nós, o centro de comunicação, a atenção primária à saúde; os pontos de atenção secundários e terciários; os sistemas de apoio; os sistemas logísticos e o sistema de governança da rede de atenção à saúde. Por fim, tem-se os modelos de atenção à saúde como terceiro elemento componente das RAS. Podem ser conceituados como os sistemas lógicos que organizam seu funcionamento, articulando de forma singular as relações entre a população e suas subpopulações estratificadas por riscos, os focos das intervenções do sistema de atenção à saúde e os diferentes tipos de intervenções sanitárias, definidos a partir da análise situacional de saúde.,11

Outrossim, ações podem ser empreendidas visando o sucesso na implementação das RAS. Nesse contexto, é possível destacar a disponibilidade de profissionais com formação para atuar na comunidade e com utilização das melhores evidências científicas na terapêutica dos problemas mais prevalentes; as ações de saúde abrangentes e articuladas contemplando vigilância, prevenção de doenças e a promoção de saúde; e o gerenciamento do cuidado para garantia da continuidade, por meio da regulação do acesso e integração com os demais níveis de atenção; disponibilidade de uma gama de serviços, incluindo outras especialidades médicas de maior prevalência quando necessário.

Evidências internacionais reforçam que as RAS oportunizam melhores resultados sanitários e econômicos dos sistemas de atenção à saúde, por exemplo, na atenção às pessoas em cuidados paliativos, ${ }^{20}$ na avaliação de admissões inapropriadas, ${ }^{21}$ na saúde do idoso, ${ }^{21}$ e na saúde 
Redes de atenção à saúde: uma estratégia para integração dos sistemas de saúde $\mid 8$

mental. ${ }^{22}$ Portanto, a construção de uma rede ampla de cuidados em saúde, implica em considerar o nível de atenção, em razão da especificidade dos serviços, a organização por especialidade por ciclo de vida ou outros critérios com base em diagnóstico situacional.

\section{Cenário das Redes de Atenção no Brasil}

No SUS, a modelagem de redes regionais de atenção à saúde é favorecida atualmente pelo Pacto de Gestão, que tem por objetivo melhorar a eficácia e a qualidade da Gestão no SUS, com a definição de metas sanitárias. Para isso, deverá integrar as ações de promoção da saúde, da atenção básica à saúde, da atenção especializada ambulatorial e hospitalar, da vigilância em saúde e da gestão do trabalho e educação na saúde nas mudanças do modelo de atenção à saúde, visando a regionalização. ${ }^{23}$

Esse processo exige a cooperação entre os municípios de determinada região de saúde, além da qualificação da APS como instância organizadora do sistema e coordenadora do cuidado ofertado. Assim, todos os componentes das RAS mostram-se importantes e diferenciam-se pelas respectivas densidades tecnológicas, uma vez que a concepção de hierarquia é substituída pela de poliarquia e o sistema organiza-se sob a forma de uma rede horizontal de atenção à saúde, tendo a APS no centro. ${ }^{4,11} \mathrm{O}$ sistema de referência e contrarreferência, por exemplo, é considerado como um dos elementos-chave para esta organização. A utilização de um formulário de referência e contrarreferência tem por objetivo repassar informações úteis que contribuam com a qualidade da atenção e comunicação entre os profissionais.

Oficialmente no SUS existem dois marcos legais para as RAS. A Portaria nำ 4.279, de 30 de dezembro de 2010 que estabelece diretrizes para a organização das redes de atenção à saúde no âmbito do SUS e o Decreto no 7.508, de 28 de junho de 2011, que define a RAS como uma forma de organização das ações e serviços de promoção, prevenção e recuperação da saúde integrados por meio de sistemas de apoio técnico, logístico e de gestão com a finalidade de garantir a integralidade do cuidado..$^{5,6,11}$ 
Desse modo, após a pactuação entre o Ministério da Saúde, os estados e os municípios, buscou-se a consolidação conceitual e os pontos de propostas operacionais para a implantação de redes temáticas nas regiões de saúde do Brasil. ${ }^{5}$ Em um momento inicial, estipulou-se as RAS como política estruturante do SUS, sendo discutidas com estados e municípios as áreas a serem priorizadas em razão do perfil epidemiológico e assistencial. Os esforços foram concomitantes ao desejo de ampliar o escopo da APS, no sentido de torná-la resolutiva, por meio do fortalecimento do trabalho em equipe, ampliação e valorização das ações dos profissionais.

Posteriormente, respeitando as especificidades de cada rede temática, foram pensadas as fases para o processo de implantação: diagnóstico (análise situacional) e adesão (política e técnica com definição do grupo condutor); desenho da rede (pactuação dos fluxos, dos pontos de atenção e suas missões); contratualização dos pontos de atenção; qualificação dos elementos da rede e certificação da rede. $^{7}$ Ressalta-se que a maior eficácia das ações de saúde se dá pela constituição de relações mais horizontalizadas, com cuidados múltiplos pautados em uma integração sistêmica entre os diversos serviços, que passam a se comportar como pontos de atenção inter-relacionados e com canais permanentes de comunicação. ${ }^{19}$

Desse modo, a atuação nestas ações, acompanhada do gerenciamento do cuidado clínico o qual é primordial para a consolidação das RAS, requer que a participação dos profissionais supere modelos verticalizados de atenção, contemplando a participação social, gerencial e administrativa. No Brasil, foram identificados estudos com foco na implementação de redes de atenção materno-infantil, puerpério, acompanhamento do crescimento e desenvolvimento das crianças, ${ }^{24}$ no planejamento de ações das Unidades de Pronto Atendimento (UPA) na rede de serviços de saúde. ${ }^{25}$ Nesse sentido, as redes regionalizadas e integradas de atenção à saúde oferecem uma condição estruturalmente mais adequada para efetivação da integralidade da atenção e reduzem os custos dos serviços por conferir uma maior racionalidade sistêmica na utilização dos recursos. 
Ainda que regulamentada, o desafio em consolidar as RAS no SUS esbarra na alocação de recursos para atender às necessidades assistenciais, na relação entre as esferas de governo e suas atribuições no contexto da descentralização da saúde, na gestão intergovernamental e na integração em um modelo assistencial no qual a APS tenha centralidade. ${ }^{10,16}$ Entende-se que o modelo de financiamento burocrático e fragmentado, é incompatível para viabilizar ações planejadas, programadas e pactuadas que contemplem os elementos conceituais das RAS. Para assegurar esta proposta, faz-se necessário a criação de mecanismos formais de contratualização, entre reguladores e os prestadores de serviços, na programação entre serviços e ações nos diferentes níveis de atenção.

\section{Conclusões}

Este artigo proporcionou um estudo teórico reflexivo sobre as RAS e seus conceitos teóricos e marcos legais nas políticas de saúde. Incluíram-se os conceitos de integração dos serviços de saúde, o histórico e os elementos conceituais das RAS. A integração de serviços e sistemas de saúde tem impacto positivo na qualidade de vida dos pacientes e suas famílias, garantindo cuidado contínuo e integral, melhorando a adesão ao tratamento e prevenindo o surgimento de agravos de saúde e eventos adversos. Foram elencadas experiências internacionais e os eixos prioritários, no Brasil, com suas aplicações. A RAS tem se demonstrado como uma estratégia à integração dos serviços de saúde, oportunizando uma condição indispensável para a qualificação e a continuidade do cuidado. Entende-se que a RAS é primordial na superação de lacunas assistenciais, na racionalização e otimização dos recursos disponíveis.

Os desafios para a consolidação da proposta estão no papel central dos serviços de APS e, sobretudo, no modelo de financiamento. A produção acerca das RAS no contexto do SUS, ainda pouco desenvolvida, pode ser fator limitante do alcance das reflexões apresentadas neste artigo. 
As considerações apresentadas podem colaborar para a disseminação de evidências relevantes para o fortalecimento da Atenção Primária à Saúde.

\section{Referências}

1. Chueiri OS, Harzheim E, Gauche H, Vasconcelos LLC. Pessoas com doenças crônicas, as redes de atenção e a Atenção Primária à Saúde. Divulg Saúde Debate [Internet]. 2014 [acesso em 2019 fev 14];52:114-24. Disponível em: http://cebes.org.br/site/wp-content/uploads/2014/12/Divulgacao-52.pdf

2. Organização Mundial da Saúde (OMS). Declaração de Alma-Ata. In: Conferência Internacional sobre Cuidados Primários de Saúde; 1978 ; Alma-Ata, Cazaquistão; 1978. p. 6-12.

3. Bainbrige D, Brazil K, Krueger P, Ploeg J, Taniguchi A, Darnay J. Measuring horizontal integration among health care providers in the community: an examination of a collaborative process within a palliative care network. J Interprof Care [Internet]. 2015 [acesso em 2019 fev 14];3(29):245-52. Disponível em: https://doi.org/10.3109/13561820.2014.984019

4. Mendes EV. As redes de atenção à saúde [Internet]. Brasília (DF): Organização Panamericana da Saúde; 2011 [acesso em 2019 fev 14]. Disponível em: https://www.paho.org/bra/index.php?option=com_docman\&view=download\&category_slug=servicossaude-095\&alias=1402-as-redes-atencao-a-saude-2a-edicao-2\&Itemid=965

5. Brasil. Ministério da Saúde. Portaria n 4.279, de 30 de dezembro de 2010. Estabelece diretrizes para a organização da Rede de Atenção à saúde no âmbito do sistema único de saúde [Internet]. Brasília (DF);

2010 [acesso em 2019 fev 14]. Disponível em: http://conselho.saude.gov.br/ultimas_noticias/2011/img/07_jan_portaria4279_301210.pdf

6. Brasil. Ministério da Saúde. Decreto n 7.508, de 28 de junho de 2011. Regulamenta a Lei no 8.080, de 19 de setembro de 1990, para dispor sobre a organização do Sistema Único de Saúde - SUS, o planejamento da saúde, a assistência à saúde e a articulação interfederativa, e dá outras providências [Internet]. Brasília (DF); 2011 [acesso em 2019 fev 14]. Disponível em: http://www.planalto.gov.br/ccivil_03/_Ato2011-2014/2011/Decreto/D7508.htm

7. Magalhães Júnior HM. Redes de Atenção à Saúde: rumo à integralidade. Divulg Saúde Debate [Internet]. 2014 [acesso em 2019 fev 14];52:15-37. Disponível em: http://cebes.org.br/site/wpcontent/uploads/2014/12/Divulgacao-52.pdf

8. Santos CM, Barbieri AR, Gonçalves CCM, Tsuha DH Avaliação da rede de atenção ao portador de hipertensão arterial: estudo de uma região de saúde. Cad Saúde Pública [Internet]. 2017 [acesso em 2019 fev 14];33(5):e00052816. Disponível em: http://www.scielo.br/pdf/csp/v33n5/1678-4464-csp-33-05e00052816.pdf 
Redes de atenção à saúde: uma estratégia para integração dos sistemas de saúde | 12

9. Organização Mundial da Saúde (OMS). WHO global strategy on people-centred and integrated health services: interim report [Internet]. Geneva: World Health Organization; 2015 [acesso em 2019 fev 14]. Disponível em: http://www.who.int/iris/handle/10665/155002

10. Santos AM, Giovanella L. Regional governance: strategies and disputes in health region management. Rev Saúde Pública [Internet]. 2014 [acesso em 2019 fev 14];48(4):622-31. Disponível em: http://www.scielo.br/pdf/rsp/v48n4/0034-8910-rsp-48-4-0622.pdf

11. Mendes EV. As redes de atenção à saúde. Ciênc Saúde Colet [Internet]. 2010 [acesso em 2019 fev 14];15(5):2297-305. Disponível em: http://www.scielo.br/pdf/csc/v15n5/v15n5a05.pdf

12. Armitage GD, Suter E, Oelke ND, Adair CE. Health systems integration: state of the evidence. Int J Integr Care [Internet]. 2009 [acesso em 2019 fev 14];9(82):1-11. Disponível em: https://www.ijic.org/articles/10.5334/ijic.316/

13. Goodwin N, Smith J. The evidence base for integrated care [Internet]. London: The King's Fund and the Nuffield Trust; 2012 [acesso em 2019 fev 14]. Disponível em: https://www.ijic.org/articles/10.5334/ijic.316/

14. Suter E, Oelke ND, Adair CE, Armitage GD. Ten key principles for successful health systems integration. Healthc Q [Internet]. 2009 [acesso em $2019 \mathrm{fev}$ 14];13(N Spec):16-23. Disponível em: https://www.ncbi.nlm.nih.gov/pmc/articles/PMC3004930/pdf/nihms1308.pdf

15. Organização Panamericana de Saúde (OPAS). Informe Dawson sobre el futuro de los servicios medicos y afines, 1920 [Internet]. Ministerio de Salud de la Gran Bretana. Londres: Organização Panamericana de Saúde; 1964 [acesso em 2019 fev 14]. Disponível em: http://apps.who.int/iris/bitstream/handle/10665/169356/42178.pdf?sequence=1\&isAllowed=y

16. Kuschnir R, Chorny AH. Redes de atenção à saúde: contextualizando o debate. Ciênc Saúde Colet [Internet]. 2010 [acesso em 2019 fev 14];15(5):2307-16. Disponível em: http://www.scielo.br/pdf/csc/v15n5/v15n5a06.pdf

17. Silva RM, Andrade LOM. Coordenação dos cuidados em saúde no Brasil: o desafio federal de fortalecer a atenção primária à saúde. Physis (Rio J) [Internet]. 2014 [acesso em 2019 fev 14];4(24):1207-28. Disponível em: http://www.scielo.br/pdf/physis/v24n4/0103-7331-physis-24-04-01207.pdf

18. Almeida PF, Santos AM, Santos VP, Silveira Filho RM. Integração assistencial em região de saúde: paradoxo entre necessidades regionais e interesses locais. Saúde Soc [Internet]. 2016 [acesso em 2019 fev 14];25(2):320-35. Disponível em: http://www.scielo.br/pdf/sausoc/v25n2/1984-0470-sausoc-25-02-00320.pdf

19. Medeiros KKAS, Pinto Júnior EP, Bousquat A, Medina MG. O desafio da integralidade no cuidado ao idoso, no âmbito da Atenção Primária à Saúde. Saúde Debate [Internet]. 2017[acesso em 2019 fev 14];41(N Esp 3):288-95. Disponível em: https://www.scielosp.org/pdf/sdeb/2017.v41nspe3/288-295/pt 
20. Eerden MH, Ewert B, Hodiamont F, Hesse M, Hasselaar J, Radbruch L. Towards accessible integrated palliative care: perspectives of leaders from seven European countries on facilitators, barriers and recommendations for improvement. J Integr Care (Brighton) [Internet]. 2017[acesso em $2019 \mathrm{fev}$ 14];25(3):222-32. Disponível em: https:/www.emeraldinsight.com/doi/pdfplus/10.1108/JICA-03-2017-0006

21. Mangan C, Pietroni M, Porter D. "Being brave": a case study of how an innovative peer review approach led to service improvement. J Integr Care (Brighton) [Internet]. 2016 [acesso em 2019 fev 14];24(4):201-13. Disponível em: https://www.emerald.com/insight/content/doi/10.1108/JICA-06-20160021/full/html

22. Oreilly O, Hanlon D. Irish Integrated Care Programme for Chronic Disease - supporting general practice. Int J Integr Care [Internet]. 2017 [acesso em 2019 fev 14];17(3):1-8. Disponível em: https://www.ijic.org/articles/abstract/10.5334/ijic.3167/

23. Menicucci TMG, Costa LA, Machado JA. Pacto pela saúde: aproximações e colisões na arena federativa. Ciênc Saúde Colet [Internet]. 2018 [acesso em 2019 fev 14];23(1):29-40. Disponível em: https://www.scielosp.org/pdf/csc/2018.v23n1/29-40/pt

24. Frank BRB, Toso BRGO, Viera CS3, Guimarães ATB, Caldeira S. Avaliação da implementação da Rede Mãe Paranaense em três regionais de saúde do Paraná. Saúde Debate [Internet]. 2016 [acesso em 2019 fev 14];40(109):164-74. Disponível em: http://www.scielo.br/pdf/sdeb/v40n109/0103-1104-sdeb-40-10900163.pdf

25. Uchimura LYT, Viana ALD, Silva HP, Ibanez N. Unidades de Pronto Atendimento (UPAs): características da gestão às redes de atenção no Paraná. Saúde Debate [Internet]. 2015 [acesso em 2019 fev 14];39(107):972-83. Disponível em: http:/www.scielo.br/pdf/sdeb/v39n107/0103-1104-sdeb-39-10700972.pdf

\section{Autor correspondente}

Adalvane Nobres Damaceno

E-mail: adalvanedamaceno@gmail.com

Endreço: Programa de Pós Graduação em Enfermagem - Rua: São Manoel, 963 - Bairro: Rio Branco - Porto Alegre, Brasil.

CEP: $90620-110$

\section{Contribuições de Autoria}

Autor 1 - Adalvane Nobres Damaceno

Idealizador do manuscrito, redação e revisor do texto final.

Autor 2 - Maria Alice Dias da Silva Lima 
Redes de atenção à saúde: uma estratégia para integração dos sistemas de saúde | 14

Orientadora do manuscrito e revisora do texto final.

Autor 3 - Vanessa Rodrigues Pucci

Revisão crítica do manuscrito.

Autor 4 - Teresinha Heck Weiller

Revisão crítica do manuscrito.

\section{Como citar este artigo}

Damaceno AN, Lima MADS, Pucci VR, Weiller TH. Redes de atenção à saúde: uma estratégia para integração dos sistemas de saúde. Rev. Enferm. UFSM. 2020 [Acesso em: Anos Mês Dia]; vol.10 e14: 1-14. DOI:https://doi.org/10.5902/2179769236832 